\title{
353. Qualitätssicherung durch EDV-Basisdokumentation in der Chirurgie
}

\author{
W. Stock, H. Mackrodt, Th. Stangl und K. Dresing \\ Chirurgische Klinik, Marien-Hospital (Chefarzt: Prof. Dr. W. Stock), Akademisches Lehrkrankenhaus, \\ Rochusstraße 2, D-4000 Düsseldorf
}

\section{Checking of Results in the Surgical Clinic by EDP-Controlled Basic Documentation}

\begin{abstract}
Summary. EDP-controlled basic documentation is essential for the checking of results in the surgical clinic. Diagnosis, method of operation, length of hospitalization, and administrative data such as age, confession, martital status etc. are registered. Using the ICD and VESKA keys, the facts are fed into the computer. We have now developed a key of our own, which is fed in simultaneously. When this information has been recorded, the data can then be compared using tables. The procedure is presented using the example "complications following resections of colon and rectum", dependent upon age group.
\end{abstract}

Key words: Checking of results in surgical treatment - Clinical documentation - Computer technology.

Zusammenfassung. Die EDV-Basisdokumentation stellt eine Voraussetzung für die Qualitätssicherung in der Chirurgie dar. Diagnose, Operationsverfahren, Liegedauer und die Verwaltungsdaten wie Alter, Religion, Familienstand etc. werden mit ihr erfaßt. Die Codierung der Daten erfolgt mit dem ICD- und VESKASchlüssel. Zur Erfassung der postoperativen Komplikationen wurde ein eigener Komplikationsschlüssel erstellt. Nach Eingabe in den Computer können mit Kreuztabellen z. B. Behandlungsergebnisse, Operationsverfahren, Patientenalter, Liegedauer und Tumorerkrankungen miteinander verglichen werden. Das Verfahren wird mit dem Beispiel „Komplikationen nach colorectalen Resektionen” in Abhängigkeit der Altersgruppen vorgestellt.

Schliisselwörter: Qualitätssicherung in der Chirurgie - Klinikdokumentation - EDV - Technik.

\section{Experimentelle Untersuchungen zur Einheilung zementfreier Schaftendoprothesen mit sogenannter madreporischer Oberfläche}

\author{
H. Rechl, R. Ascherl, W. Erhardt, M.-L. Schmeller, K. Geißdörfer, G. Rupp ${ }^{1}$, R. Senekowitsch, \\ H. Kriegel ${ }^{2}$ und G. Blümel \\ Institut für Experimentelle Chirurgie (Dir.: Prof. Dr. G. Blümel) der Technischen Universität München, \\ Ismaninger Straße 22, D-8000 München 80, ${ }^{1}$ Unfallchir. Abteilung Landeskrankenhaus Vöcklabruck \\ (Vorstand: Prim. Dr. G. Rupp) und ${ }^{2}$ Abteilung für Nuklearbiologie (Dir.: Prof. Dr. H. Kriegel) der \\ Gesellschaft für Strahlen- und Umweltforschung mbH München-Neuherberg
}

\section{Experimental Study on the Healing of Cementless Femoral Endoprostheses With a So-called Madrepore-like Surface}

Summary. In the implantation without cement of total endoprostheses, implants of different surface reliefs have been used more frequently. In adult dogs $(n=9)$ total replacement of the left hip has been performed using an implant, the femoral compartment of which had a so-called madrepore-like surface. In the radiological followups as well as in histological and microangiographic investigations 6 months after operation an ingrowth of bone with only a small interface could be seen even between the balls of the madrepore-like surface, whereas smooth surfaces showed a broad interface of connective tissue. Scintigrams indicate that bone healing around the implants is complete $10-15$ weeks after the operation.

Key words: Total endoprosthesis - Ball coated surface - Bony ingrowth.

Zusammenfassung. Bei der zementfreien Implantation von Totalendoprothesen finden Implantate mit unterschiedlichen Oberflächen reliefs vermehrt Anwendung. An erwachsenen Hunden $(n=9)$ wurde linksseitig ein Hüftgelenkersatz durchgeführt, dessen femorales Kompartment mit sog. madreporischer Oberfläche versehen war. In radiologischen Verlaufskontrollen sowie histologischen und mikroangiographischen Untersuchungen nach 6 Monaten zeigt sich ein grenzschichtarmes Einwachsen des Knochens an den Prothesenoberflächen und in den Kugelzwischenräumen, wohingegen glatte Vergleichsoberflächen von einem breiten bindegewebigem Saum umgeben sind. Szintigraphisch erscheinen die Einheilungsvorgänge nach 10-15 Wochen abgeschlossen.

Schlïsselwörter: Totalendoprothesen - Madreporische Oberfläche - Knochenheilung. 